\title{
Silicon-Organic Hybrid (SOH) Frequency Comb Source for Data Transmission at $784 \mathrm{Gbit} / \mathrm{s}$
}

\author{
C. Weimann ${ }^{1}$, S. Wolf ${ }^{1}$, D. Korn ${ }^{1}$, R. Palmer ${ }^{1}$, S. Koeber ${ }^{1}$, R. Schmogrow ${ }^{1}$, P. C. Schindler ${ }^{1}$, \\ L. Alloatti ${ }^{1}$, A. Ludwig ${ }^{1}$, W. Heni ${ }^{1}$, D. Bekele ${ }^{1}$, D. L. Elder ${ }^{2}$, H. Yu ${ }^{3}$, W. Bogaerts ${ }^{3}$, L. R. Dalton ${ }^{2}$, \\ W. Freude ${ }^{1}$, J. Leuthold ${ }^{1,4}$, C. Koos ${ }^{1}$ \\ ${ }^{(1)}$ Karlsruhe Institute of Technology (KIT), Institutes IPQ and IMT, 76131 Karlsruhe, Germany, \\ (2) University of Washington, Department of Chemistry, Seattle, WA 98195-1700, United States \\ ${ }^{(3)}$ Ghent University - IMEC, Photonics Research Group, Gent, Belgium \\ ${ }^{(4)}$ Electromagnetic Fields \& Microwave Electronics Laboratory (IFH), ETH-Zurich, Zurich, Switzerland \\ claudius.weimann@kit.edu, christian.koos@kit.edu
}

\begin{abstract}
We demonstrate a frequency comb generator using silicon-organic hybrid (SOH) electrooptic modulators to obtain flat-top comb spectra. This is the first demonstration of a modulator-based frequency comb generator on silicon. The viability of the device is confirmed in a data transmission experiment achieving an aggregate data rate of $784 \mathrm{Gbit/s}$.
\end{abstract}

\section{Introduction}

Frequency combs enable a wide range of attractive applications such as optical metrology, arbitrary-waveform generation ${ }^{1}$ or high-speed data communications at $\mathrm{Tbit} / \mathrm{s}$ aggregate transmission rates ${ }^{2}$. A particularly versatile and flexible approach to generate frequency combs is based on modulation of a continuous-wave (cw) laser with an appropriate arrangement of electro-optic phase shifters, each of which is driven with a periodic signal of distinct amplitude and phase $^{3}$. This results in a precisely adjustable line spacing, tunable center wavelength, and narrow linewidth. A key figure of the associated phase shifters is the achievable modulation bandwidth and the modulation depth. Experimental demonstrations have been performed on the basis of conventional lithium niobate devices ${ }^{4}$ or InPbased photonic integrated circuits ${ }^{5}$. For advanced transmitter systems, it would be highly desirable to co-integrate frequency comb generators with the wealth of devices offered by silicon photonics. However, electro-optic modulation on silicon currently relies on depletion-type pn phase shifters. These devices are characterized by a low efficiency, which impedes broadband frequency comb generation. In this paper we demonstrate a novel concept for silicon-based comb generators that are based on silicon organic hybrid $(\mathrm{SOH})$ integration ${ }^{6}$. The $\mathrm{SOH}$ approach combines silicon nanophotonic waveguides with organic cladding materials to realize highly efficient broadband phase shifters. Using a single dualdrive Mach-Zehnder interferometer (MZI) modulator with a $U_{\pi} L$ product of $2 \mathrm{~V} \mathrm{~mm}$, we demonstrate generation of 7 lines within a power range of $2 \mathrm{~dB}$. The device enables data transmission of $28 \mathrm{GBd}$ QPSK signals on 7 adjacent carriers. Using polarization multiplexing, this results in an aggregate rate of $784 \mathrm{Gbit} / \mathrm{s}$. This is to the best of our knowledge the first demonstration of a modulator-based frequency comb source on silicon.

\section{Silicon-organic hybrid (SOH) frequency comb generator}

The frequency comb generator consists of a dual drive MZI modulator exploiting $\mathrm{SOH}$ phase modulators $^{6,7}$ as depicted in Fig. 1(a). The phase modulator consists of a slot waveguide electrically connected to a coplanar transmission line through $60 \mathrm{~nm}$ thick doped silicon strips. The waveguide is covered with the electro-optic chromophore DLD164, which entirely fills the slot. The voltage applied to the transmission line drops across the narrow slot, resulting in a high electric field that strongly overlaps with the optical mode. Silicon waveguides are fabricated in IMEC by $193 \mathrm{~nm}$ deep-UV lithography on an SOI wafer with a $2 \mu \mathrm{m}$ thick buried oxide and a $220 \mathrm{~nm}$ thick device layer. The dual drive MachZehnder modulator (MZM), Fig. 1(b), consists of two identical $2 \mathrm{~mm}$ long phase-modulators. The phase modulators are driven individually by two coplanar copper slotlines. The electro-optic cladding is applied by spin coating and poled at elevated temperatures by applying a DC voltage across the slotline electrodes. The voltagelength product of each phase modulator was measured at DC to be $U_{\pi} L=2 \mathrm{Vmm}$. The experimental setup for comb generation is depicted in Fig.1(b): The high bandwidth and low $U_{\pi} L$ product of the phase shifters allow for efficient generation of higher-order optical 

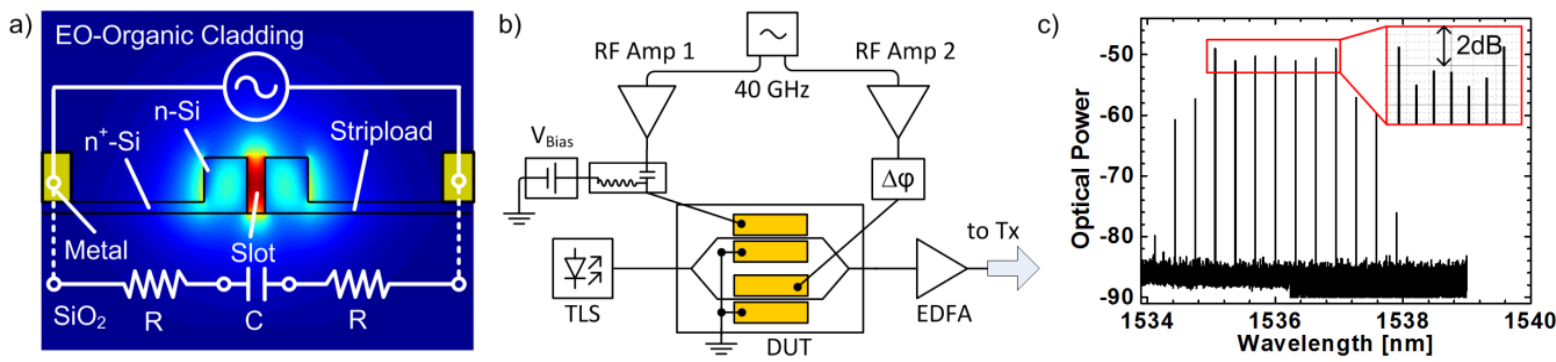

Fig. 1: a) Schematic and simulated optical mode of a silicon-organic hybrid (SOH) phase-modulator. The two rails of a silicon slot waveguide are electrically connected to metal electrodes by $60 \mathrm{~nm}$ high $n$-doped silicon slabs. The waveguide is covered by an electro-optic cladding material (DLD164), which entirely fills the slot. b) Integrated frequency comb generator: A tunable laser source (TLS) is coupled to a dual-drive $\mathrm{SOH} \mathrm{Mach}$ Zehnder modulator (MZM) via grating couplers. The arms are driven by two sinusoidal $40 \mathrm{GHz}$ signals. Flat combs are obtained by carefully adjusting the signal powers and phases along with the bias voltage $V_{\text {Bias. }}$ Fiberchip coupling losses are compensated by an erbium-doped fiber amplifier (EDFA). c) Flat-top spectrum obtained for electrical drive powers of $28 \mathrm{dBm}$ and $23 \mathrm{dBm}$, showing 7 lines within $2 \mathrm{~dB}$ flatness. The lines are spaced by $40 \mathrm{GHz}$.

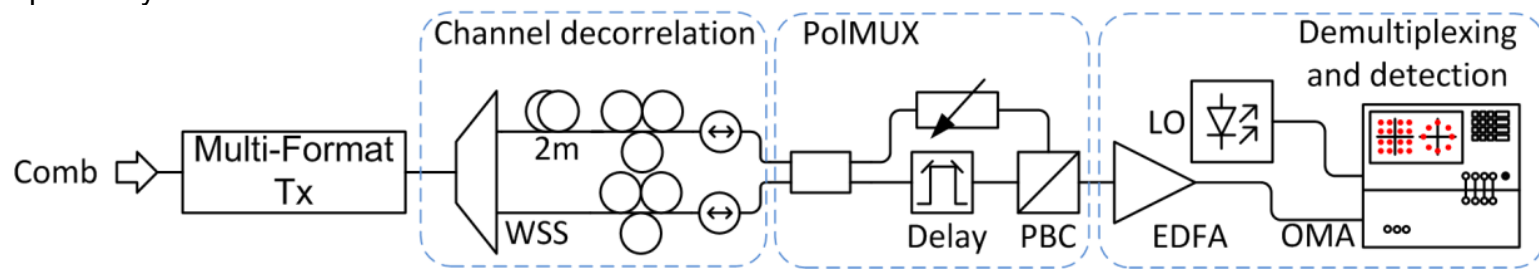

Fig. 2: Data transmission setup: Each comb line is modulated with a 28 GBd QPSK signal. Even and odd channels are separated with a wavelength-selective switch (WSS) to decorrelate neighboring channels. Both data streams are merged and are polarization multiplexed (PoIMUX). After amplification, the channels are demultiplexed and detected by an optical modulation analyzer (OMA) and a tunable laser acting as a local oscillator (LO).

sidebands, see Fig. 1c). By carefully adjusting the drive parameters ${ }^{3}$, we achieve a spectral flatness of 7 lines within $2 \mathrm{~dB}$. The line spacing amounts to $40 \mathrm{GHz}$.

\section{WDM data transmission}

We have performed a proof-of-principle data transmission experiment using the setup depicted in Fig. 2. We use a single Multi-Format transmitter to modulate each line with the same QPSK NRZ signal at a symbol rate of $28 \mathrm{GBd}$. For decorrelation of the data signals, even and odd channels are separated by a wavelengthselective switch (Finisar WaveShaper, WSS), delayed with respect to each other, and recombined in a fiber-based $2 \times 2$ coupler. Polarization division multiplexing (PDM) is emulated in a similar manner by splitting the data stream, delaying one part with respect to the other, and recombining them in a polarization beam combiner.

To characterize the signal quality at the receiver side, we use an optical modulation analyzer (OMA, Agilent N4391A) along with a tunable laser acting as a local oscillator. Digital signal processing is performed for polarization demultiplexing and equalization. We record the constellation diagrams and extract the error vector magnitude (EVM) as a measure for signal quality, Fig. 3. Note that the comb generator used for the data transmission experiment had a slightly worse performance and a higher voltage-length product of $U_{\pi} L=3.2 \mathrm{~V} \mathrm{~mm}$ than the device discussed in the previous section. As a consequence, the spectral flatness of the comb was worse with a power variations of $7 \mathrm{~dB}$ for 7 spectral lines. In addition, we used $28 \mathrm{GBd}$ NRZ QPSK and fairly narrow carrier spacing of $40 \mathrm{GHz}$. This leads to an overlap of the data spectra and hence to significant inter-channel interference (ICl) and signal quality impairments. The different channels show EVM values between $20.7 \%$ and $37.0 \%$ and measured bit error ratios (BER) are better than $4 \times 10^{-3}$, which is still below the hard-decision FEC threshold ${ }^{8}$ of $4.5 \times 10^{-3}$. Using $28 \mathrm{GBd}$ PDM-QPSK signals on 7 adjacent carriers we hence obtain a gross aggregate data rate of $784 \mathrm{Gbit} / \mathrm{s}$. In future experiments, $\mathrm{ICl}$ can be avoided by using advanced multiplexing schemes such as OFDM or band-limited Nyquist pulse shaping techniques. This will enable chip-scale multichannel transceivers providing aggregate data rates beyond $1 \mathrm{Tbit} / \mathrm{s}$. 


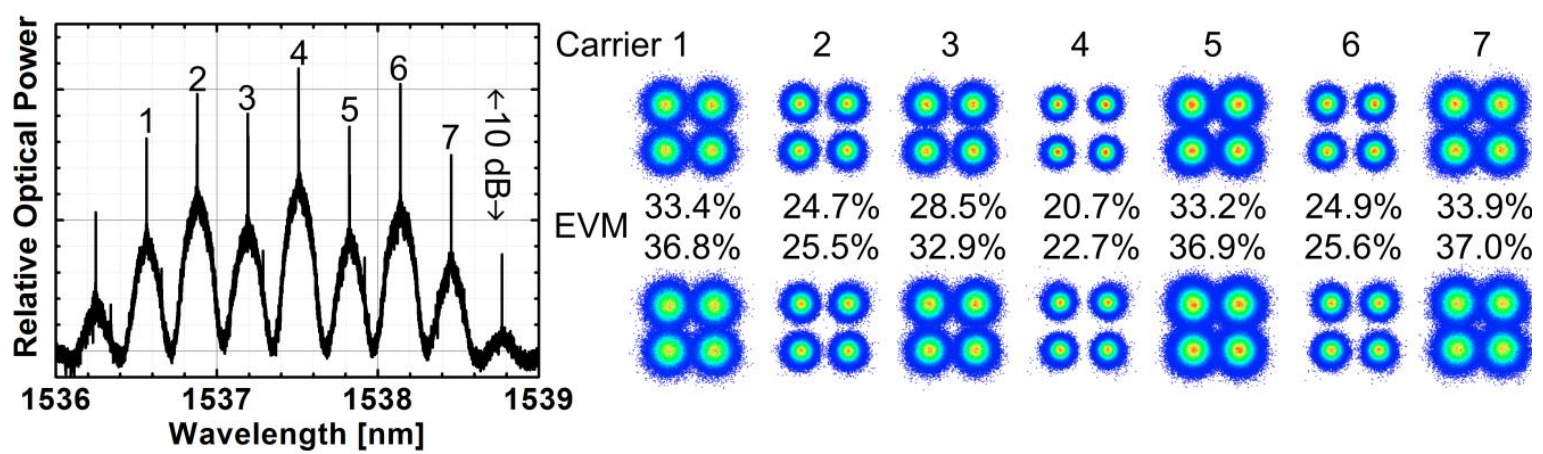

Fig. 3: Data transmission results: Data transmission of $784 \mathrm{Gbit} / \mathrm{s}$ using QPSK signals on 7 carriers generated by an integrated $\mathrm{SOH}$ comb source. Comb spectrum (left) and constellation diagrams for all channels and both polarization are depicted along with measured EVM values. BER below hard-decision threshold are achieved for all signals.

\section{Conclusions}

We have demonstrated a modulator-based frequency comb generator based on siliconorganic hybrid (SOH) integration. The device can generate flat-top frequency comb spectra with 7 lines within a $2 \mathrm{~dB}$ power range. Data transmission was demonstrated with gross data rates of $784 \mathrm{Gbit} / \mathrm{s}$ and $\mathrm{BER}$ values below harddecision FEC.

\section{Acknowledgements}

This work was supported by the European Research Council (ERC Starting Grant 'EnTeraPIC', number 280145), by the Karlsruhe Nano-Micro Facility (KNMF), by the Karlsruhe School of Optics \& Photonics (KSOP), by the Helmholtz International Research School for Teratronics (HIRST), by the Initiative and Networking Fund of the Helmholtz Association, by the EU-FP7 projects SOFI, OTONES, and
PHOXTROT and the Alfried Krupp von Bohlen und Halbach Foundation.

\section{References}

[1] S. T. Cundiff et al., Nat. Photon., vol. 4, no. 11, pp. 760-766, (2010).

[2] D. Hillerkuss, et al., Nat. Photon., vol. 5, no. 6, pp. 364-371, (2011)

[3] T. Sakamoto et al., Opt. Lett., Vol. 32, No. 11,1515 (2007).

[4] I. Gheorma et al., Photon. Tech. Lett., vol. 19, no. 13, pp. 1011-1013, (2007).

[5] N. Dupuis, et al., J. Lightwave Tech., vol. 30, no. 4, pp. 466-472, (2012).

[6] L. Alloatti, et al., Opt. Express, 19(12):11841-11851, (2011).

[7] R. Palmer, et al., Photonics Journal, v.5(2), p.6600907, April (2013)

[8] F. Chang, et al., IEEE Commun. Mag, vol. 48, no. 3, pp. S48, (2010). 